\title{
Left atrium mixoma on Marshall's ligament: an unusual or uncommon representative
}

\section{Mixoma de atrio izquierdo sobre el ligamento de Marshall: un representativo inusual o infrecuente}

\section{Gutenberg Navarro-Zambrano*, Samuel Ramírez-Marroquín, Felipe Santibáñez-Escobar, and Guillermo Castro-Lozano}

Department of Cardiothoracic Surgery, Instituto Nacional de Cardiología Ignacio Chávez, Faculty of Medicine, Universidad Nacional Autónoma de México, Mexico City, Mexico

Atrial myxoma is a primary cardiac tumor, common and benign, which is associated with complications that will depend on its location'. Left atrial myxomas are more common than those with right location (5:1); $85 \%$ are located on the left atrial septum, $11 \%$ on the right atrial septum and $1 \%$ are multiple 2.3

An uncommon case of myxoma, not reported in the literature, is that located on Marshall's ligament, which entails high risk of post-embolization ischemic stroke, can have clinical manifestations and should be considered in differential diagnosis for its detection, identification and timely treatment. The case of a 64-year-old female patient with a medical history of supraventricular tachycardia, clinical data consistent with transient ischemic attack, and coronary computed tomography angiography incidental findings showing the presence of a tumor (myxoma) or thrombus in the left atrium is presented. Transesophageal echocardiogram documented a tumor in the left atrium suggestive of myxoma (22 x $23 \mathrm{~mm})$, which was heterogeneous, had defined borders, was slightly mobile, located on Marshall's ligament, close to the left atrial appendage (Fig. 1). Surgical treatment was decided by resection via median sternotomy and left atriotomy. The findings were: $2.4 \mathrm{x}$ 2.4-mm left atrial tumor adhered to Marshall's ligament (Fig. 2). Histopathological examination diagnosed a myxoma. The patient evolved favorably with 24-hour stay in the intensive care unit and hospital discharge at six days.

\section{Funding}

None.

\section{Conflicts of interest}

None.

\section{Ethical disclosures}

Protection of human and animal subjects. The authors declare that no experiments have been performed on humans or animals for this investigation. DOI: 10.24875/ACME.M21000228 2604-7063 / @ 2020 Instituto Nacional de Cardiología Ignacio Chávez. Published by Permanyer. This is an open access article under the CC BY-NC-ND license (http://creativecommons.org/licenses/by-nc-nd/4.0/).
Available online: $20-10-2021$ Arch Cardiol Mex (Eng). 2021;91(3):330-331 www.archivoscardiologia.com 


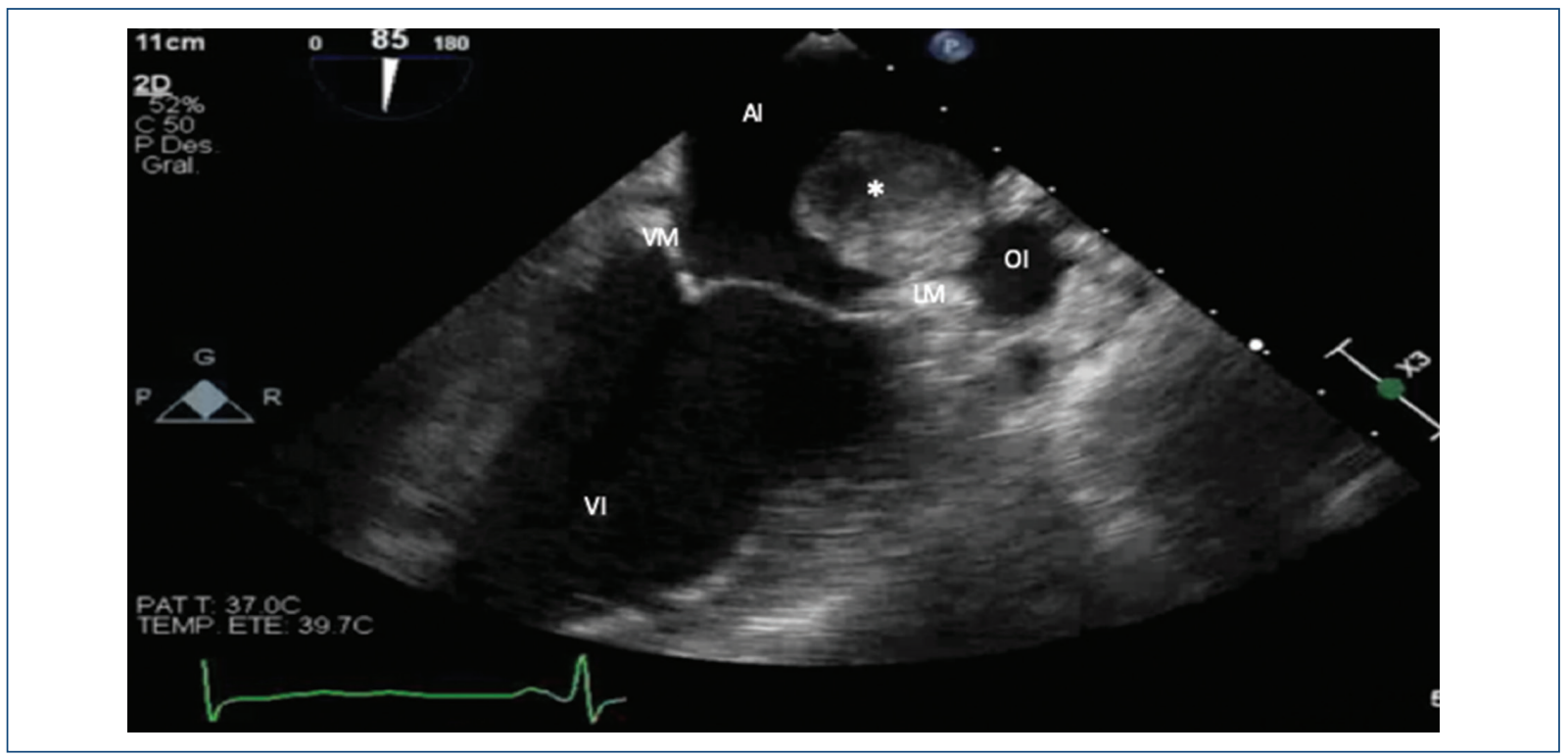

Figure 1. Transesophageal echocardiogram showing a $22 \times 23 \mathrm{~mm}$ mass in the left atrium located on Marshall's ligament (asterisk), consistent with myxoma. Al: left atrium; LM: Marshall's ligament; VI: left ventricle.

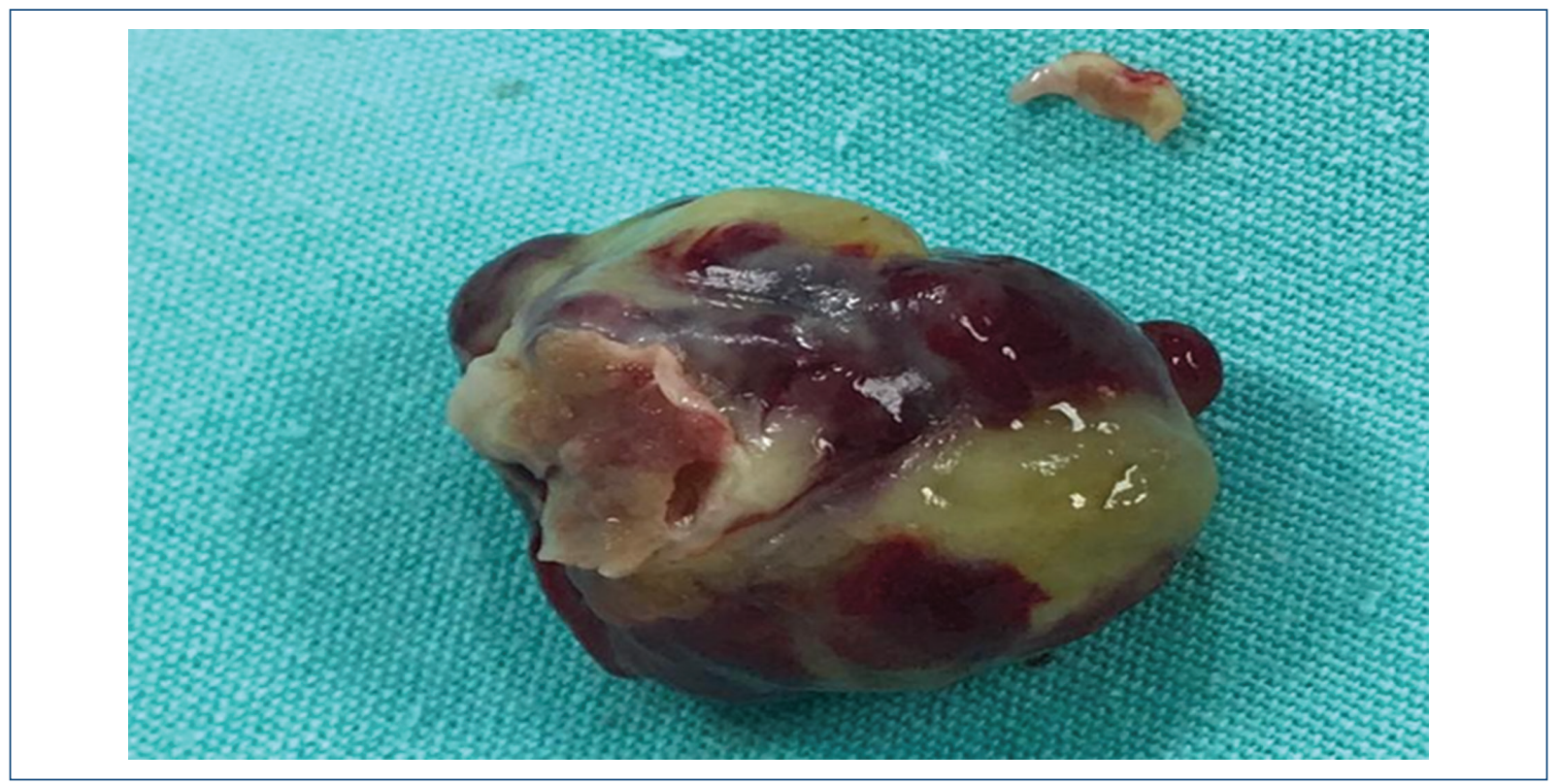

Figure 2. Gross view of the $24 \times 24-\mathrm{mm}$ left atrial myxoma, located on Marshall's ligament.

Confidentiality of data. The authors declare that they have followed the protocols of their work center on the publication of patient data.

Right to privacy and informed consent. The authors declare that no patient data appear in this article.

\section{References}

1. Burgos E, Bermúdez J, Chávez W. Mixoma auricular relacionado con evento cerebrovascular vascular isquémico. Med Clin (Barc). 2018;151: e69-e70.

2. Insen Z, Hela M, Khadija M, Zouhayer J. Cerebral embolism complicating left atrial myxoma: a case report. Pan Afr Med J. 2016;24:140.

3. Yin W, Xian F, Xiao L, Xinmin Z. Stroke and peripheral embolisms in a pediatric patient with giant atrial myxoma: case report and review of current literature. Medicine (Balt). 2018;97:e11653. 